\title{
A Calibration-Free Methodology for Resonantly Enhanced Photoacoustic Spectroscopy using Quantum Cascade Lasers
}

\author{
Metin Ilke, Ralf Bauer, Member, IEEE, and Michael Lengden, Member, IEEE
}

\begin{abstract}
Photoacoustic spectroscopy (PAS) is a highly sensitive technique for trace gas sensing, which requires frequent re-calibration for changing environmental influences and input light power fluctuation. This is a major drawback against its deployment for on-site, long-term remote applications. To address this drawback here we present the theory and application of a Calibration-Free Wavelength Modulation Photoacoustic Spectroscopy (CF-WM-PAS) technique. It is applied for measurements of $\mathrm{CH}_{4}$ gas concentration in the mid-infrared at $8.6 \mu \mathrm{m}$ wavelength using a Quantum Cascade Laser (QCL). The method normalizes the second harmonic $\left(R_{2 f}\right)$ component, dominated by laser-gas interaction and optical intensity, by the first harmonic $\left(R_{1 f}\right)$ component dominated by Residual Amplitude Modulation (RAM) DC offset, to isolate the output from changes in the gas matrix, optical intensity and electrical gain. This normalization technique removes influences from changes in the resonant frequency, gas concentration and incident optical power. It is confirmed using a $\pm 1600 \mathrm{~Hz}$ change in modulation frequency around the resonance, a $1 \%$ to $10 \%$ change in gas concentration and an up to $78.3 \%$ attenuation in input light intensity with a custom built miniaturized 3D-printed sensor. A Normalized Noise Equivalent Absorption (NNEA) of $4.85 \times 10^{-9} \mathrm{Wcm}^{-1} \mathrm{~Hz}^{-1 / 2}$ for calibration-free $R_{2 f} / R_{1 f}$ measurements is demonstrated.
\end{abstract}

Index Terms-Photoacoustic spectroscopy, gas sensing, miniaturized, calibration free, Quantum cascade lasers, 3D printing.

\section{INTRODUCTION}

$\mathbf{T}$ Here has been a significant increase in photoacoustic spectroscopy (PAS) research in recent years, targeting the development of sensors for high sensitivity gas species concentration measurement. This new interest in PAS is due to a combination of factors. The first is the maturity of midinfrared (mid-IR) quantum cascade lasers (QCLs) to highly stable, high power devices [1] [2] [3] [4] [5]. The use of midinfrared sources for spectroscopic gas measurements allows optical access of the fundamental rotational-vibrational excitation levels of gas species, which can have 10-100 times higher absorption cross-section than higher order harmonic rotationalvibrational transitions accessible with near-infrared devices [6]. The second is the reduced cost of PAS, as compared to optical absorption sensing techniques such as tunable diode

Manuscript received October 16, 2019. This work was supported in part by the EPSRC under Grant EP/P001661/1.

M. Ilke, R. Bauer, and M. Lengden are with the Centre for Microsystems and Photonics, Department of Electronic and Electrical Engineering, University of Strathclyde, Glasgow, G1 1XW, U.K. (e-mail: metin.ilke@strath.ac.uk; ralf.bauer@strath.ac.uk; michael.lengden@strath. ac.uk).

Data can be accessed via: Digital Object Identifier https://doi.org/10.15129/e4f9103e-256c-42a7-9bc9-44a8227a4f22 laser absorption spectroscopy (TDLAS). In TLDAS the cost of both a mid-IR optical source and receiver can make sensor development prohibitive. However, the laser-gas interaction in PAS is measured indirectly via a pressure wave, generated through localised heating and cooling in a periodically excited gas sample [7]. Therefore, the photo-receiver can be replaced by a cheap, small-scale acoustic transducer. Thirdly, as both PAS and TDLAS measurement sensitivities are linearly proportional to the input optical power, and photo-receivers are limited to powers less than $1 \mathrm{~mW}$, the use of higher power lasers in PAS systems can achieve higher overall measurement sensitivities [6].

PAS systems can be operated in similar manner to TDLAS with wavelength modulation (TDLAS-WM), where high frequency signal generation and demodulation using lockin amplification dramatically improves the overall SNR [8]. In WM spectroscopy, a dual current modulation is applied to the laser, with a slow frequency ramp waveform to scan over the targeted rotational-vibrational transition and a higherfrequency sinusoidal modulation that generates laser-gas interaction signals at the harmonics of the modulation frequency. There is, however, a subtle difference in the signals generated by the two techniques. In TDLAS-WM, the dominant signal output at the fundamental modulation frequency (1f) is known as the residual amplitude modulation (RAM). This RAM component is generated by the direct intensity modulation (IM) output from the laser, and is present even when no laser-gas interaction occurs [9]. However, in PAS there is no inherent RAM signal, as the lasers IM does not produce any localized heating in the absence of target gas absorption, resulting in an intrinsically zero-background measurement technique at $1 \mathrm{f}$, often seen as an advantage in PAS with wavelength modulation (PAS-WM).

The use of PAS-WM is commonly combined with resonant miniaturized structures, such as resonant cell designs [10] and resonant acoustic transducers [11], most commonly quartz tuning forks (QTFs). Recently, a resonance-based PAS technique has been presented that has a three-fold enhancement through the production of an optical grating that moves at the speed of sound through the gas [12]. One key disadvantage of these resonant PAS measurements is the requirement for calibration of the cell resonance, which varies as a function of gas density. As with all PAS systems there is also a requirement to calibrate for the input optical power and the acoustic transducer responsivity.

A number of techniques have been proposed to ensure 
accurate calibration in PAS; intermittent scanning of the modulation frequency to measure the cell's resonant frequency [13], continual measurement of the temperature [14] and multifrequency excitation [15]. Frequent characterisation of the resonance by sequentially recording the PAS signal amplitude as a function of frequency is the most basic technique to give an accurate measurement of the resonance frequency and quality factor. The frequency scan can be performed by either varying the laser's modulation frequency or the frequency applied to a miniaturized speaker placed into the resonator [13]. However, this method takes a significant period of time to acquire data and it cannot account for short-term changes in cell temperature and system pressure. For faster response, the use of a short square pulse [16] or chirped modulation [17] allows the excitation of multiple frequencies simultaneously. By normalization of the Fourier transform of the acoustic response by the laser's multiple drive frequencies the resonance curve can be determined. However, this method only works with high Q-factor resonators and when the PAS signal amplitudes are relatively high. In addition, the technique becomes more complex for changes in system parameters, such as temperature and pressure. Active pressure and temperature tracking and acoustic compensation algorithms can be used to estimate the shift in the resonant frequency and Qfactor after the measurement [18] [19]. However, these methods generally require extensive pre-deployment calibration. Another calibration methodology monitors the phase of the generated PAS signal using phase sensitive detection via a lock-in amplifier (LIA) and therefore tracks changes in the resonant frequency, as the signal at resonance should be inphase with the laser drive signal [20] [21]. However, this technique does not compensate for any changes in sensor performance i.e. acoustic transducer output drift. A technique specifically developed for calibration in QTF enhanced PAS is beat frequency quartz enhanced PAS (BF-QEPAS), where the modulation frequency is slightly shifted from the resonance frequency and the laser wavelength is modulated with a high frequency modulation, compared to the QTF response time [22]. The Q-factor, resonance frequency and target gas concentration can be identified by analysing the beat signal between the laser modulation and the instantaneous QTF resonance frequency. However, this technique is highly challenging to be applied in conventional PAS systems with lower response time, frequency and Q-factor.

In this paper, we report on the development, performance characteristics and underlying theory of a calibration-free 3Dprinted PAS gas sensor, utilizing a continuous wave QCL at a wavelength of $8.625 \mu \mathrm{m}$. The proposed methodology is based on the $2 \mathrm{f} / 1 \mathrm{f}$ calibration approach used for TDLAS-WM [23]. In $2 \mathrm{f} / 1 \mathrm{f}$, the total magnitude of the first harmonic, $R_{1 f}$, whose major component is the RAM DC offset, is used to normalise the total magnitude of the signal recovered at the second harmonic, $R_{2 f}$, which is both gas and optical intensity dependent but typically has zero RAM. This division removes any intensity dependence as all components in the final signal are proportional to either the ratio of the amplitude of sinusoidal intensity modulation $(\Delta I)$ to the input intensity $(I)$, $\Delta I / I$, or its inverse, which is a constant for any given laser operational parameter. Furthermore, this technique removes any dependence on signal gain as both the recovered $R_{1 f}$ and $R_{2 f}$ have the same overall gain. In this newly proposed PAS technique a signal similar to the RAM signal is used for normalisation. However, in this case the first harmonic DC offset is generated by optical absorption into the polymer used to construct the resonant cell.

\section{CF-PAS-WMS SIGNAL GENERATION}

The gas cell used in this work is based on a longitudinal acoustic resonance design [10]. The amplified acoustic signal, generated by the laser-gas interaction within the resonant chamber of the longitudinal resonator, $S_{r}\left(\omega_{r}\right)$, can be described by the equation [10]:

$$
S_{r}\left(\omega_{r}\right)=\frac{\left(\gamma_{a}-1\right) P_{g-a b s} K_{m}\left(\omega_{r}\right) Q F}{V \omega_{r}}
$$

where $\omega_{r}=2 \pi f_{r}$ is the angular resonance frequency, $\gamma_{a}$ is the adiabatic constant, $K_{m}\left(\omega_{r}\right)$ describes the microphone response at $\omega_{r}, Q$ is the quality factor of the resonator, $V$ is the volume of the gas chamber, $\mathrm{F}$ is the optical excitationresonator overlap integral and $P_{g-a b s}$ is total optical power absorbed by the gas and can be calculated using the BeerLambert law,

$$
P_{g-a b s}=P_{L}\left(1-e^{-S(T) \phi(\nu, T, p) p \chi l}\right)
$$

where $P_{L}$ is the incident optical power, $T$ is the gas temperature, $S$ and $\phi$ are the line-strength and line-shape function of the targeted spectral feature respectively, $\nu$ is the optical frequency, $p$ is the cell pressure, $\chi$ is the target species mole fraction, and $l$ is the laser-gas interaction path length inside the resonator.

If there is significant incident optical power absorbed by the side walls of the resonant chamber of the PAS cell, a separate acoustic signal can be generated. This cell dependent absorption signal, CDAS - $S_{s}\left(\omega_{r}\right)$, is assumed to be due to the rapid heating and cooling of the boundary layer of the gas on the surface of the solid material, and is amplified by the resonator in a similar manner to the gas absorption signal, and can be described by the equation:

$$
S_{s}\left(\omega_{r}\right)=\frac{P_{s} K_{m}\left(\omega_{r}\right) Q A_{s} l F}{V \omega_{r}}
$$

where $P_{s}$ refers to the optical power incident on the solid and $A_{s}$ is the probability of absorption by the polymer. The polymer in this work is a broadband absorber at the target wavelength. However, the particular polymer used in this work may not be suitable to generate a CDAS at other wavelengths, particularly in the near-infrared or visible wavelengths where limited absorption will occur.

To generate the acoustic signals in a resonant cell the incident optical power is modulated at the PAS cell's resonant frequency in the form $P_{L}=P_{0}\left(\nu_{0}\right)+\Delta P \cos \left(\omega_{r} t\right)$. This modulated optical power can be substituted into equations 1 and 2 to give:

$$
S_{r}\left(\omega_{r}\right)=G\left[P_{0}(\nu)+\Delta P \cos \left(\omega_{r} t\right)\right]\left[1-e^{-k l}\right]
$$


where $G$ is the overall gain of the system including resonant amplification and microphone response, $P_{0}(\nu)$ is the laser power output at line centre, $\Delta \mathrm{P}$ is the power modulation amplitude and $k=S(T) \phi(\nu, T, p) p \chi$.

The laser's wavelength/frequency modulated output also needs to be considered, given as $\nu=\nu_{0}+\delta \nu \cos \left(\omega_{r} t+\psi\right)$. The modulated wavelength interaction with the gas can be used to generate a Fourier series expansion of the form:

$$
1-e^{-k l}=1-\sum_{k=0}^{k=\infty} H_{k}(\nu, \delta \nu) \cos \left(k\left(\omega_{r} t-\psi\right)\right)
$$

where $\delta \nu$ is the frequency modulation (FM) amplitude, $\psi$ is the phase delay of the frequency modulation with respect to the intensity modulation (FM/IM), and $H_{k}$ are the Fourier coefficients described by Rieker et al. [23].

Substituting equation (5) into (4) gives the final expression for the gas generated photoacoustic signal as:

$$
\begin{aligned}
S_{r}\left(\omega_{r}\right)= & G\left[P_{0}(\nu)+\Delta P \cos \left(\omega_{r} t\right)\right] \\
& {\left[H_{0}^{\prime}-H_{1} \cos \left(\omega_{r} t-\psi\right)-H_{2} \cos \left(2 \omega_{r} t-2 \psi\right)\right.} \\
& \left.-H_{3} \cos \left(3 \omega_{r} t-3 \psi\right) \ldots \ldots\right]
\end{aligned}
$$

where $H_{0}^{\prime}=1-H_{0}$.

Equation 6 can be further extended by including an IM term that describes the optical absorption by the solid polymer using equation 3 , resulting in a final microphone output signal of the form:

$$
\begin{aligned}
S_{r}\left(\omega_{r}\right)= & G\left[P_{0}(\nu)+\Delta P \cos \left(\omega_{r} t\right)\right] \\
& {\left[H_{0}^{\prime}-H_{1} \cos \left(\omega_{r} t-\psi\right)-H_{2} \cos \left(2 \omega_{r} t-2 \psi\right)\right.} \\
& \left.-H_{3} \cos \left(3 \omega_{r} t-3 \psi\right) \ldots . .\right] \\
& +G\left[P_{s}+\Delta P_{s} \cos \left(\omega_{r} t\right)\right] A_{s} l
\end{aligned}
$$

We have assumed that there is negligible FM signal generated through absorption by the solid, due to the broadband absorbing nature of the polymer.

The microphone output, described by equation 7 , is then passed to a lock-in amplifier (LIA). For the calibration-free PAS technique we present here we need to consider the LIA output for two different operational conditions. The first operational condition is defined as the first harmonic recovery condition. In this case, the laser current modulation frequency and the LIA reference frequency are both set to be equal to the resonant frequency of the cell, $\omega_{r}$. For simplicity, we have also defined the LIA reference phase to be $\theta_{r e f}=0$. The inphase and quadrature LIA outputs for the first harmonic are then given as:

$$
\begin{gathered}
X_{1 f}=G \Delta P H_{0}^{\prime}-G P_{0} \cos (\psi) H_{1} \\
-\frac{G \Delta P}{2} \cos (2 \psi) H_{2}+G \Delta P_{s} A_{s} l \\
Y_{1 f}=-G P_{0} \sin (\psi) H_{1}-\frac{G \Delta P}{2} \sin (2 \psi) H_{2}
\end{gathered}
$$

The second operational condition is defined as the second harmonic condition. In this case, the laser current is modulated at half the cell's resonant frequency, $\omega_{r} / 2$, but the LIA reference frequency is locked to $\omega_{r}$. For simplicity, the LIA reference phase is again set to $\theta_{r e f}=0$, giving in-phase and quadrature outputs of the LIA as:

$$
\begin{aligned}
X_{2 f}= & -G H_{2} P_{0} \cos (2 \psi) \\
& -\frac{G \Delta P}{2}\left[H_{1} \cos (\psi)+H_{3} \cos (3 \psi)\right] \\
Y_{2 f}= & -G H_{2} P_{0} \sin (2 \psi) \\
& -\frac{G \Delta P}{2}\left[H_{1} \sin (\psi)+H_{3} \sin (3 \psi)\right]
\end{aligned}
$$

The most interesting feature of these LIA outputs is the term $G \Delta P_{s} A_{s} l$ in equation (8), which is the DC offset generated by optical absorption into the polymer. This DC offset, called CDAS, is similar to the RAM signal generated in TDLS-WM that is used for normalisation of optical power and signal gain in the $2 \mathrm{f} / 1 \mathrm{f}$ technique.

If we consider the case where the CDAS signal is the most dominant term i.e. there is significantly more optical power absorbed by the solid cell than by the target gas, then the magnitude of the first harmonic, $R_{1 f}\left(\omega_{r}\right)$, and the second harmonic, $R_{2 f}\left(\omega_{r}\right)$ tend to the values,

$$
R_{1 f}\left(\omega_{r}\right)=G \Delta P_{s} A_{s} l
$$

and assuming $\Delta P / 2<<P_{0}$ and $\omega_{m}=\omega_{r} / 2$,

$$
R_{2 f}\left(\omega_{r}\right)=\mathrm{GH}_{2} P_{0}
$$

The division of equation 13 by equation 12 results in a final output of:

$$
\frac{R_{2 f}\left(\omega_{r}\right)}{R_{1 f}\left(\omega_{r}\right)}=\frac{G H_{2} P_{0}}{G \Delta P_{s} A_{s} l}=\frac{H_{2} P_{0}}{\Delta P_{s} A_{s} l}
$$

As can be seen in equation 14, all dependence on the resonance frequency, microphone response and system gain is removed from the final signal. It is also clear that there is still a dependence on the gas absorption, and also on the ratio of the input optical power to the optical power absorbed by the cell. This ratio of powers can be considered a constant. However, it may be prone to long-term drift without careful consideration of cell design. Furthermore, the laser has to be modulated at two different frequencies, and $R_{1 f}\left(\omega_{r}\right)$ and $R_{2 f}\left(\omega_{r}\right)$ cannot be recovered simultaneously. Instead, they have to be recovered alternately as described in the experimental setup section III. Therefore, this normalisation technique for PAS does not have the same common mode noise cancellation advantage of TDLS-WM 2f/1f. However, the technique provides a suitable calibration for resonant PAS systems, making it possible to compensate for varying temperature and pressure conditions and both degradation in laser and acoustic transducer performance, and also allows measurement of gas concentrations over a wide range with varying gas densities without the requirement to monitor the variation in the resonant frequency. 


\section{EXPERIMENTAL SETUP}

The 3D printed gas cell is based around a design originally used for near-infrared absorption measurements [24] [25]. In this work, the near-infrared fibre collimation system has been replaced with two $0.5 \mathrm{~mm}$ thick, $6 \mathrm{~mm}$ diameter $\mathrm{CaF}_{2}$ windows to allow transmission of mid-IR light, as shown in figure 1 . The resonant chamber has a radius of $0.75 \mathrm{~mm}$ and a length of $16 \mathrm{~mm}$, giving an estimated fundamental longitudinal resonant frequency of $10.33 \mathrm{kHz}$ for $100 \% \mathrm{~N}_{2}$ at standard temperature and pressure. Buffer regions are located on each side of the resonator, each with a length of $\Lambda_{a} / 4$, where $\Lambda_{a}$ is the wavelength of the fundamental longitudinal acoustic mode. These buffers provide some immunity from noise generated from the gas flow into the cell and window noise generated through optical absorption. The gas cell was fabricated with HTM140 polymer, using a 3D stereolithography printer (EnvisionTec Aureus Desktop) with a $43 \mu \mathrm{m}$ X-Y resolution and $25 \mu \mathrm{m} \mathrm{Z}$ resolution. Gas inlet and outlet pipes of 1.6 $\mathrm{mm}$ diameter are attached to the sides of the buffer sections, and mass flow controllers (Bronkhorts EL-flow) are used to provide gas mixtures of controlled methane concentrations. A canister electret condenser microphone (Knowles FG-23742D36), with a quoted sensitivity of $-63 \mathrm{dBa} / 0.1 \mathrm{~Pa}$ at $1 \mathrm{kHz}$, is attached to the center of the resonator.

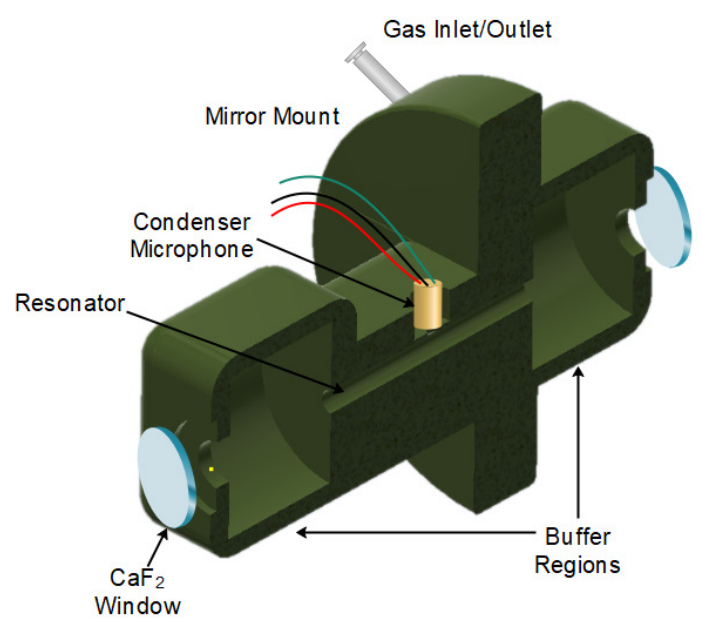

Figure 1: The schematic for the cross section of the 3Dprinted PAS cell and assembly of the other other components. The total internal cell volume is $1412 \mathrm{~mm}^{3}$.

The PAS cell is located in the experimental setup shown in the figure 2. A water cooled, continuous wave quantum cascade laser (Thorlabs-QD8650CM1), with an average power of $54 \mathrm{~mW}$, is used as the gas and polymer excitation source. The laser diode temperature is maintained at a wavelength value of $8625.5 \mathrm{~nm}$ using a Thorlabs thermoelectric controller unit (ITC4005QCL). A DC laser bias current of $700 \mathrm{~mA}$ is applied to the laser using a Thorlabs LDC 210 laser diode current controller, and a modulation current amplitude of 260 $\mathrm{mA}$ is applied for both $1 \mathrm{f}$ and $2 \mathrm{f}$ to target a modulation index of $\mathrm{m}=2.2$, the value for maximum $2 \mathrm{f}$ output [26].

In the proposed technique the laser's high frequency modulation needs to be switched between $\omega_{r}$ and $\omega_{r} / 2$ for each

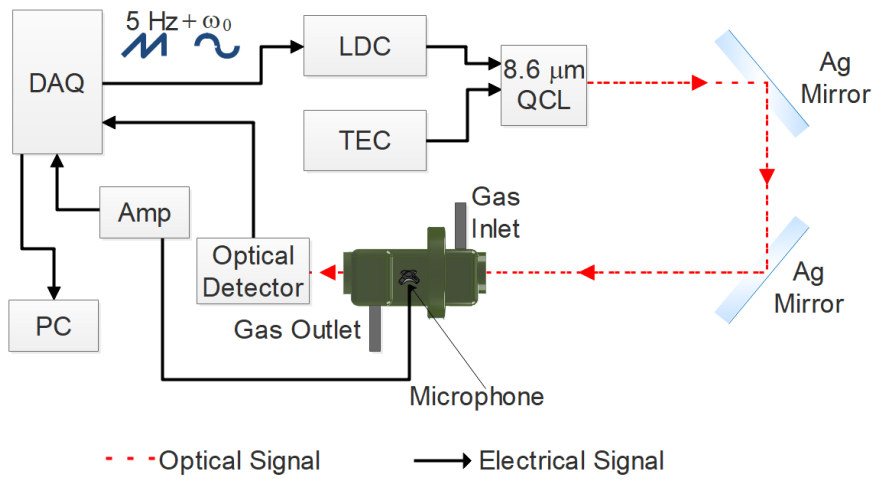

Figure 2: A schematic of the experimental setup: NI-PXI: Data acquisition/signal generation board, LDC: laser diode controller, TEC: temperature controller, Amp: low noise voltage amplifier.

alternate low frequency ramp $(5 \mathrm{~Hz})$. This signal conditioning is achieved using a bespoke NI LabVIEW code, where the output modulation drive voltages for the LDC are obtained from a 16 bit DAC via a National Instruments (NI) PXIe6361 data acquisition board (DAQ) at a sampling rate of 2 $\mathrm{MSs}^{-1}$. The generated acoustic signals are detected using the microphone, whose signal is passed through a current amplifier (Femto Messtechnik DHPCA-100) with a voltage gain of 20. The output from the amplifier is recorded using a 16 bit, $2 \mathrm{MSs}^{-1} \mathrm{ADC}$ and a digital LabVIEW lock-in amplifier is used to demodulate the recorded signals. The LIA reference signal is set to be $f_{r}$, which therefore outputs the 1 st harmonic and 2nd harmonic signal for each alternate ramp. The digital LIA was set to have a time constant of $300 \mu$ s equating to a $260 \mathrm{~Hz}$ noise bandwidth.

\section{RESUlts}

\section{A. Evidence of the Cell Dependant Absorption Signal}

The acoustic signals generated at $f_{r}=9600 \mathrm{~Hz}$ and $f_{r} / 2=$ $4800 \mathrm{~Hz}$ are shown in figures $3 \mathrm{a}$ and $3 \mathrm{~b}$ respectively, for both $10 \%$ methane in an $\mathrm{N}_{2}$ buffer gas and $100 \% \mathrm{~N}_{2}$. It is clear from figure $3 \mathrm{a}$ that there is a significant DC CDAS contribution at $R_{1 f}$ that is not due to gas absorption, but is generated by the modulation of the laser power incident on the polymer sidewall of the resonant cell. The $R_{2 f}$ signal has a very small offset contribution, due to the non-linear intensity modulation of the laser. However, the major contribution to the $R_{2 f}$ signal is the $\mathrm{H}_{2}$ component generated by the wavelength modulation interaction with the gas.

\section{B. Sensor Characterization}

Figure 4 shows the frequency characteristics of the gas cell for $R_{1 f}$ and $R_{2 f}$ using $10 \%$ methane concentration, and the resulting $R_{2 f} / R_{1 f}$ signals. It also shows data for the $R_{1 f}$ and $R_{2 f}$ signals for $100 \% \mathrm{~N}_{2}$, further highlighting the presence of CDAS in the $R_{1 f}$ signal. For these measurements the laser's modulation frequency was varied from $3 \mathrm{kHz}$ to $21.5 \mathrm{kHz}$ in $100 \mathrm{~Hz}$ increments, and the first harmonic components $X_{1 f}$ and $Y_{1 f}$, and second harmonic components, $X_{2 f}$ and $Y_{2 f}$, 


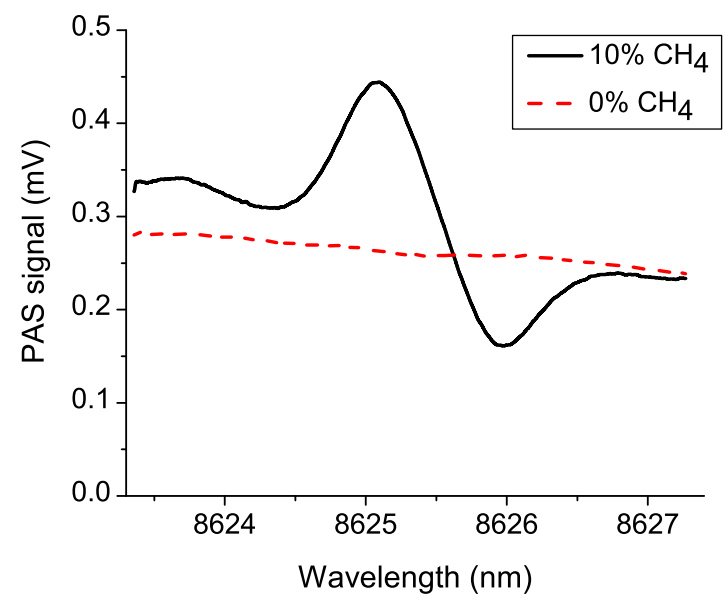

(a)

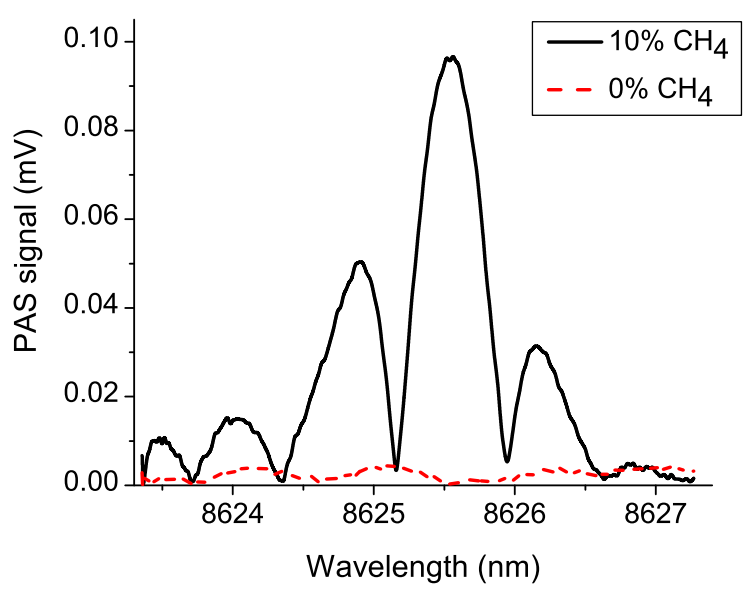

(b)

Figure 3: Magnitudes of the (a) first and (b) second harmonic components output from the LIA for both at $10 \% \mathrm{CH}_{4}$ mixed with $\mathrm{N}_{2}$ and also $0 \% \mathrm{CH}_{4}$. In (a), the DC CDAS, generated by the absorption of light by the sidewalls of the resonator, is clearly present even in the absence of any target gas. In (b), there is minimal significant non-linear CDAS present, with the most dominant signal being the $\mathrm{H}_{2}$ component due to laser-gas interaction.

were demodulated alternately using the approach described in section III. $R_{1 f}$ and $R_{2 f}$ values were then obtained, with a total of 128 measurements being averaged for each. At each frequency the LIA reference phase was adjusted to isolate the FM signal on the Y-axis of the LIA, as described in equations 8 and 9 (for more details refer to [27]), providing measurements of the amplitude of the isolated FM signal at both $\omega_{r}$ and $\omega_{r} / 2$. Finally, the $R_{2 f} / R_{1 f}$ signals were calculated for each incremental frequency.

It is clear that the $R_{2 f} / R_{1 f}$ signal is not a constant over the resonance curve, with a total percentage variation of $4.45 \%$. This implies that there will be variation in recovered concentration using this normalisation procedure if there is

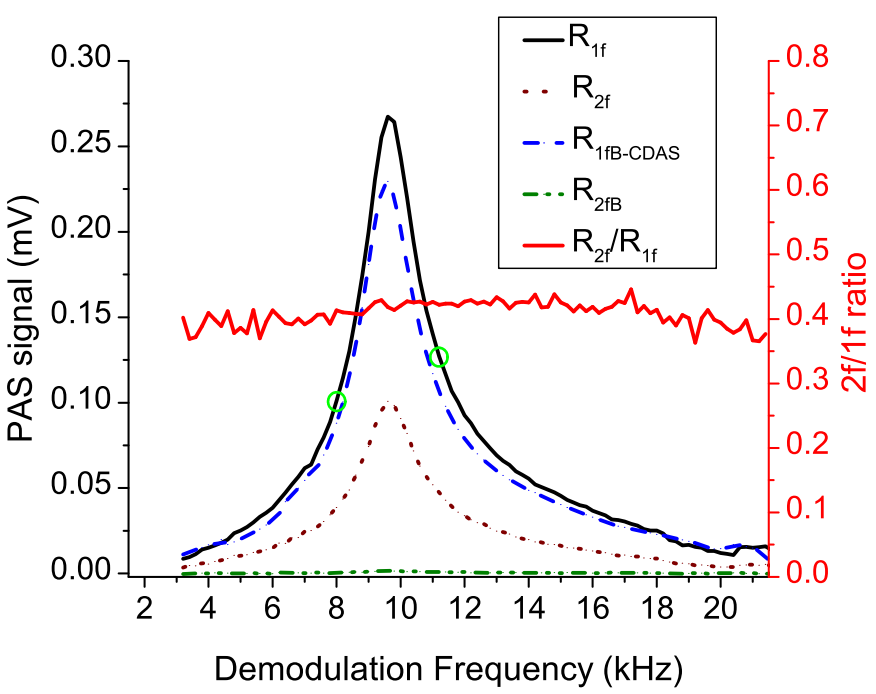

Figure 4: Frequency response profile of If peak-to-peak and $2 f$ amplitude as a function of modulation frequency for $10 \%$ $\mathrm{CH}_{4}\left(R_{1 f}\right.$ and $\left.R_{2 f}\right)$ and $0 \% \mathrm{CH}_{4}\left(R_{1 f B-C D A S}\right.$ and $\left.R_{2 f B}\right)$ concentrations. Green circles mark the $f_{r} \pm 1600 \mathrm{~Hz}$ range, indicating the range of calibration-free tests in section IV-C

variation in the overall gas density, and therefore the speed of sound in the cell. If we consider that the speed of sound for methane concentrations of 0 and $100 \%$ changes from 350 $\mathrm{ms}^{-1}$ to $448 \mathrm{~ms}^{-1}$, when considering an $N_{2}$ host gas, this change in the speed of sound will only change the resonant frequency of the resonant cell by $3.2 \mathrm{kHz}$. Therefore, if we consider a range of $\pm 1.6 \mathrm{kHz}$ from the maximum of the resonant curve in figure 4, the percentage variation in the $R_{2 f} / R_{1 f}$ signal due to the full range of methane concentrations of $0-100 \%$ is reduced to $1.45 \%$. This evidences the ability of this normalisation technique to calibrate for gas density variation, and therefore resonant frequency variation, without the requirement for any feedback.

The total magnitude of the $R_{1 f}$ signal is approximately 1.16 times larger than the generated CDAS signal. The individual contributions of $R_{1 f}$ and CDAS can, however, be controlled through the variation of the input beam shape, focus or alignment resulting in an increase or decrease of optical power incident on the side walls of the resonator. Figure 4 also shows that the amplitude of the peak $R_{2 f}$ signal at each frequency is approximately 2.6 times smaller than the peak to peak variation in the corresponding $R_{1 f}$, highlighting the reduced sensitivity of the $R_{2 f}$ signal. Furthermore, the nonlinear CDAS signal is clearly negligible, being approximately 55 times smaller than the linear CDAS signal, and therefore has very little effect on the final recovered $R_{2 f} / R_{1 f}$ signal. Finally, it is clear that the SNR of the $R_{2 f} / R_{1 f}$ reduces when operating further off resonance as the recovered $R_{2 f}$ and $R_{1 f}$ signal magnitudes reduce.

To further evidence that the same resonant acoustic mode is being excited for each of the recovered signals, the resonant magnitude profiles have been normalised to the maximum amplitude of the $R_{1 f}$ resonance profile, as shown in figure 


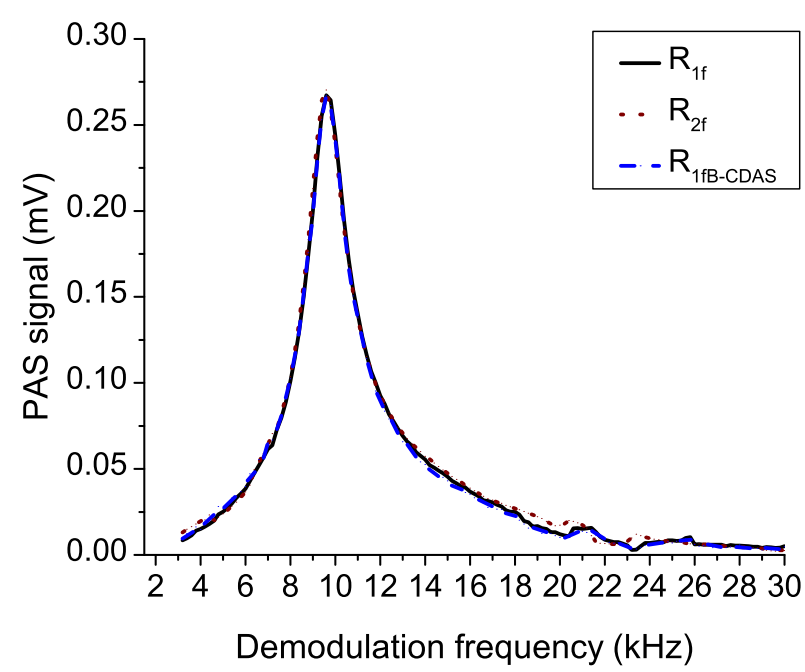

Figure 5: Resonance profile of $R_{1} f$ peak-to-peak and $R_{2} f$ amplitude as a function of modulation frequency for $10 \% \mathrm{CH}_{4}$ $\left(R_{1 f}\right.$ and $\left.R_{2 f}\right)$ and $0 \% \mathrm{CH}_{4}\left(R_{1 f B-C D A S}\right)$ gas concentrations normalized to the $R_{1 f}$ response peak

5. There is a clear overlap between the $R_{2 f}, R_{1 f}$ and linear CDAS signals. The overall Q-Factor and resonant frequency for this sensor was calculated using the data in figure 5 to be 6.6 and $9.59 \mathrm{kHz}$ respectively. The $f_{r}$ for the linear CDAS resonance is $9.49 \mathrm{kHz}$, which is $100 \mathrm{~Hz}$ lower than the $f_{r}$ of the $R_{1} f$ signal. This variation is caused by the increase in the total molar mass due to increased number of $\mathrm{N}_{2}$ molecules, leading to a reduced average speed of sound and hence a lowered resonant frequency for the CDAS signal.

\section{Validation of the CDAS Calibration Technique}

Figures $6 \mathrm{a}$ and $6 \mathrm{~b}$ show the $R_{1 f}$ and $R_{2 f}$ signals recovered at modulation frequencies of \pm 800 and $\pm 1600 \mathrm{~Hz}$ from the resonant peak, further highlighting the variation in CDAS, $R_{1 f}$ and $R_{2 f}$ as a function of modulation frequency representing a change in gas density equal to $\mathrm{CH}_{4}$ concentration from $0 \%$ to $100 \%$. The resulting $R_{2 f} / R_{1 f}$ signals are shown in figure $6 c$. As evidenced from figure $6 \mathrm{c}$ and its residual variations compared to signals at $f_{r}$, there is almost complete overlap at all frequencies.

Figure 7 shows the linearity of the $R_{2 f} / R_{1 f}$ signal as a function of methane concentration. For this data, a $10 \%$ calibrated gas mixture of $\mathrm{CH}_{4}$ with $\mathrm{N}_{2}$ (BOC - 5\% quoted supplier uncertainty) was mixed with $100 \% \mathrm{~N}_{2}$ at varying flow rates using two mass flow controllers (Bronkhorst - quoted error of $\pm 1 \%$ ), to produce concentrations ranging from $1 \%$ to $10 \%$ in $1 \%$ increments. The percentage variation of $R_{2 f} / R_{1 f}$ within a $\pm 1600 \mathrm{~Hz}$ range of the peak resonance was calculated to be $\sigma=1.45 \%$ using the data from figure 4 , which results in an estimated concentration variation of $\pm 0.12 \%$ for 0 to $100 \%$ $\mathrm{CH}_{4}$ measurement range according to the best-fit in figure 7 , further highlighting the efficacy of this technique to normalise PAS signals in-situ.

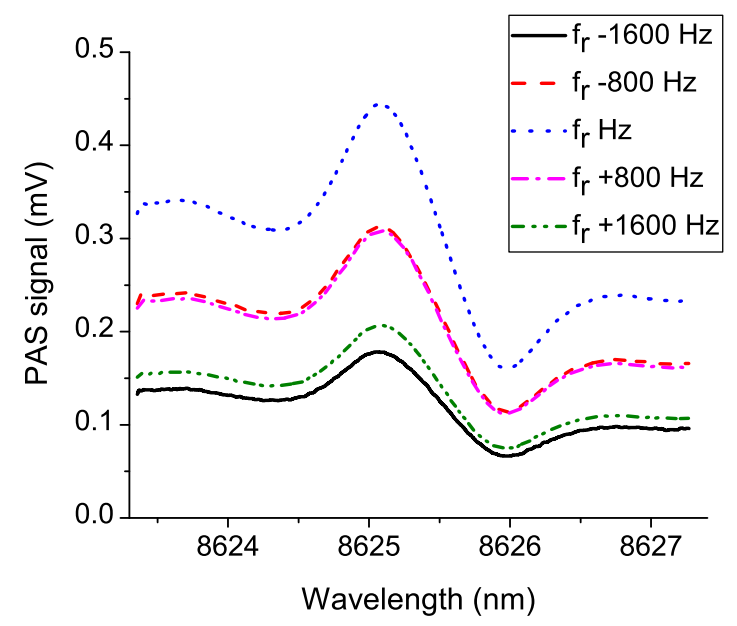

(a)

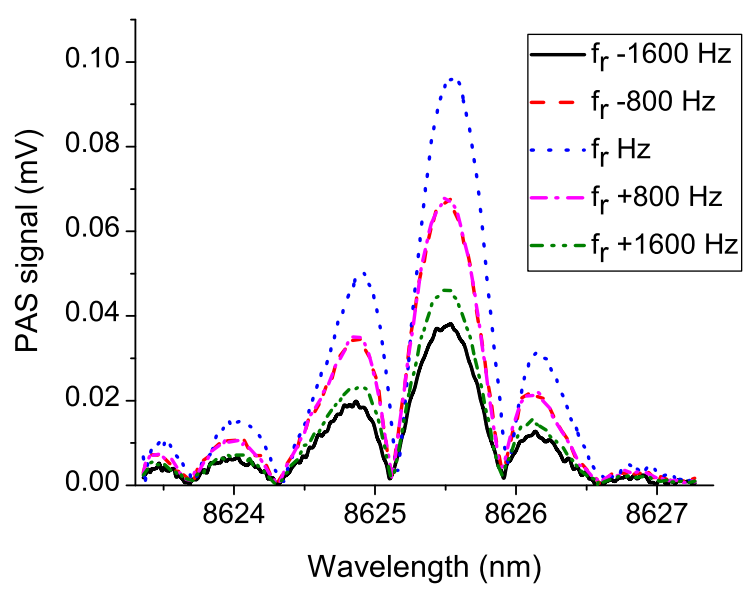

(b)

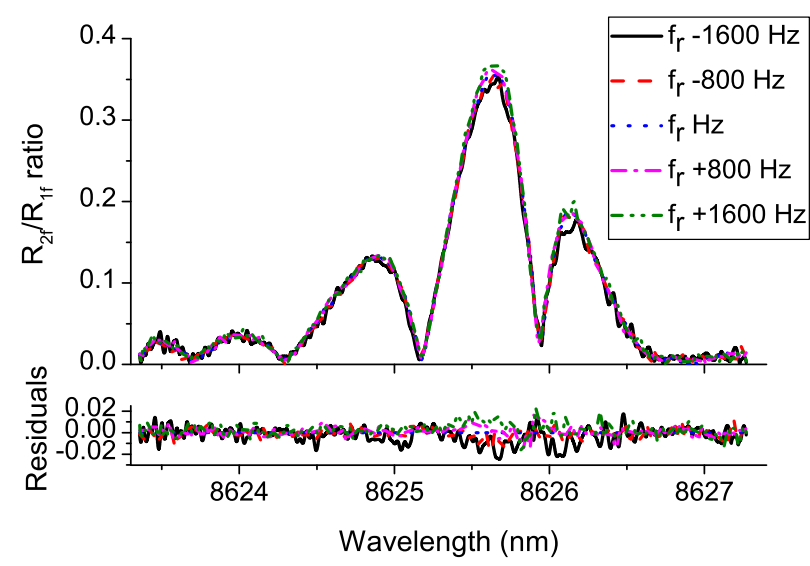

(c)

Figure 6: Generated $P A S_{W M}$ for $(a) R_{1 f}$, (b) $R_{2 f}$, and (c) $R_{2 f} / R_{1 f}$ with residuals compared to $f_{r}$, for an LIA reference frequency equal to the cell resonance, $f_{r}=9600 \mathrm{~Hz}$, and for LIA reference frequencies of $\pm 800 \mathrm{~Hz}$ and $\pm 1600 \mathrm{~Hz}$. It should be noted that the $R_{1 f}$ signals are obtained when the laser modulation frequency is equal to the LIA frequency. However, for the $R_{2 f}$ signals are obtained when the laser modulation frequency is half the LIA reference frequency. 


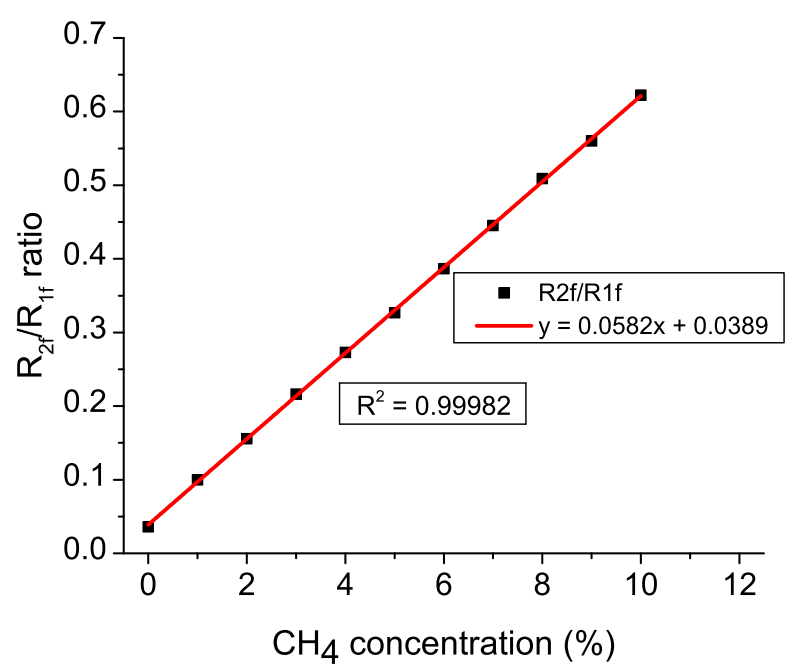

Figure 7: $R_{2 f} / R_{1 f}$ maximum signal as a function of methane concentration.

In figure $8, R_{2 f} / R_{1 f}$ signals are shown for optical power variations up to $78.3 \%$ attenuation. The input light was attenuated using acetate filters of increasing thickness. As expected, the $R_{1 f}$ signals (figure $8 \mathrm{a}$ ) and $R_{2 f}$ signals (figure $8 \mathrm{~b}$ ) reduce in magnitude as a function of optical power. However, the $R_{2 f} / R_{1 f}$ signal (figure $8 \mathrm{c}$ ) show complete overlap, and therefore shows no variation due to optical power. This highlights that this technique can also calibrate for variation in input optical power. It should be noted that normalization for power due to beam misalignment is not possible i.e. when the ratio of light interacting with the gas to the light interacting with the cell varies.

The $3 \sigma$ minimum detection limits (MDL) for $\mathrm{CH}_{4}$ gas, using laser power output at $2.466 \mathrm{~mW}$ and $16.12 \mathrm{~Hz}^{-1 / 2}$ detection bandwidth were found to be $810 \mathrm{ppm}$ for $\mathrm{R}_{1 f}, 1578$ ppm for $\mathbf{R}_{2 f}$ and 2067 ppm for $\mathbf{R}_{2 f} / \mathbf{R}_{1 f}$ measurement techniques. The Normalized Noise Equivalent Absorption (NNEA) coefficients which incorporates all system variables into one coefficient, for the used 3D-printed gas cell at the varying demodulation techniques, using a practical limit of $3 \sigma$, were found to be $1.9 \times 10^{-9} \mathrm{Wcm}^{-1} \mathrm{~Hz}^{-1 / 2}$ for $R_{1 f}, 3.71 \times 10^{-9}$ $\mathrm{Wcm}^{-1} \mathrm{~Hz}^{-1 / 2}$ for $R_{2 f}$ and $4.85 \times 10^{-9} \mathrm{Wcm}^{-1} \mathrm{~Hz}^{-1 / 2}$ for $R_{2 f} / R_{1 f}$. The NNEA values for $\mathbf{R}_{1 f}$ and $\mathbf{R}_{2 f}$ are are both superior compared to NNEA value for the $\mathrm{R}_{2 f} / \mathrm{R}_{1 f}$ technique due to combination of noise from both signals. However, this is traded off against the ability to fully calibrate the PAS signals against optical variations and cell performance. The NNEA for the $R_{2 f} / R_{1 f}$ calibration method represents a value of approximately 5.1 times that for a similar calibration technique using inter-band cascade lasers (ICL) [22]. The NNEA for $R_{2 f} / R_{1 f}$ is also a comparable value to NNEA value for fibre-based 3D-printed miniaturized PAS cells with $4.5 \times 10^{-9}$ $\mathrm{Wcm}^{-1} \mathrm{~Hz}^{-1 / 2}$ [24].

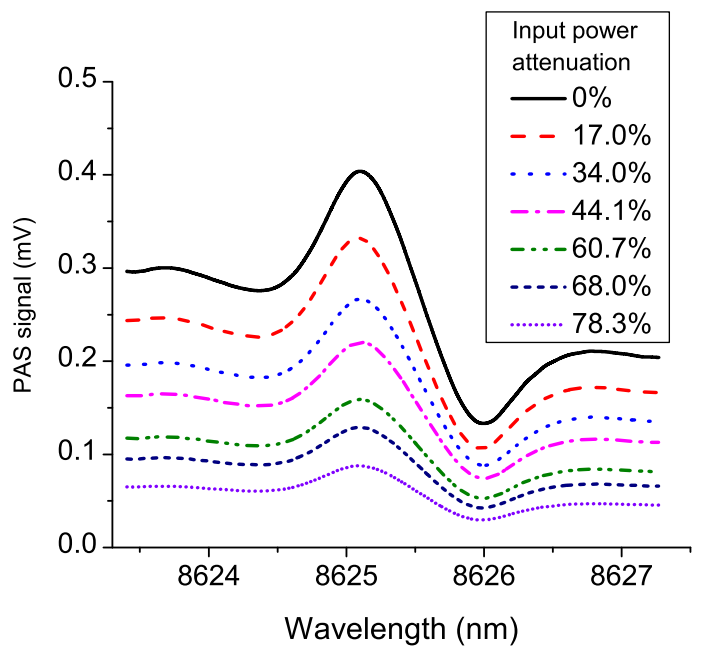

(a)

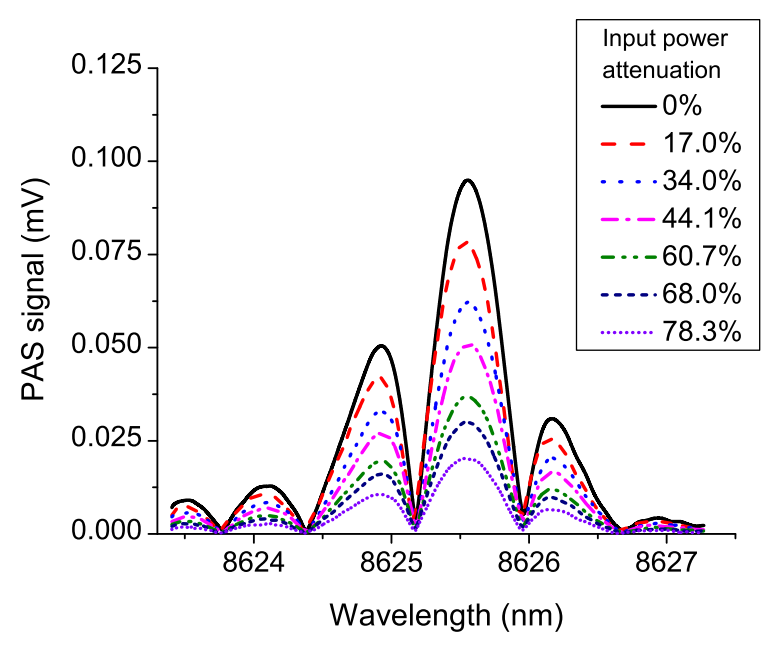

(b)

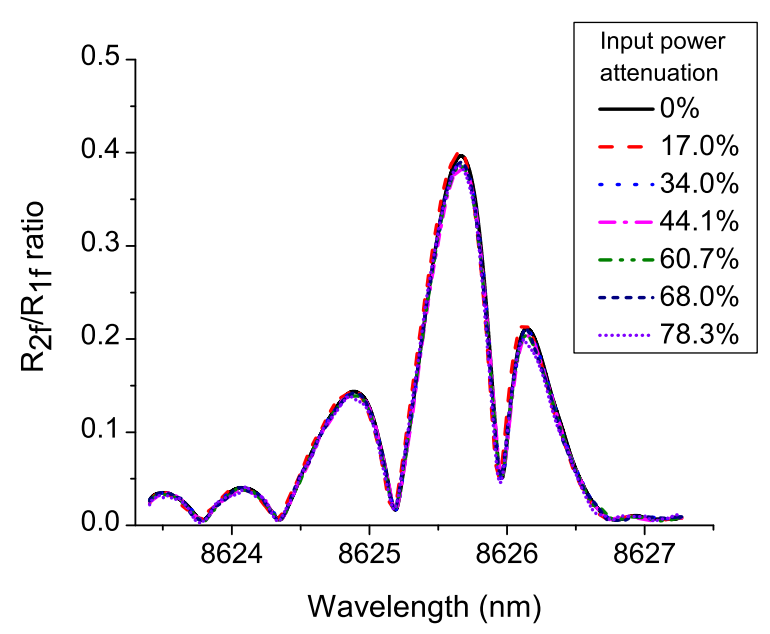

(c)

Figure 8: a) $R_{1 f}$, (b) $R_{2 f}$, and (c) $R_{2 f} / R_{1 f}$ signals obtained at varying input optical powers, showing a complete overlap of the $R_{2 f} / R_{1 f}$ signal and validating this technique for normalisation of input power variation. 


\section{DISCUSSION}

As shown in figure 7 , the PAS $R_{2 f} / R_{1 f}$ calibration technique is dependent on the absorption of optical power in the walls of the resonator, thus generating a CDAS signal. This CDAS signal provides a real-time calibration methodology that can normalise for cell performance degradation, variation in pressure and humidity and also the incident optical power. It can also be used to measure species concentrations at varying densities, without requiring tracking of the resonant frequency. However, it must be made clear that normalisation for optical power is dependent on the alignment between the cell and the incident light not drifting as a function of time. The development of a 3D printed polymer sensor that can be directly aligned and fixed to the QCL front facet would eliminate any significant optical drift, reducing signal variations through alignment changes over time.

In this paper, the main target gas was $\mathrm{CH}_{4}$ which was used instead of $\mathrm{SO}_{2}$ gas due to its similar spectral features in the interrogated wavelength range and ease of use. The MDL values given for $\mathrm{CH}_{4}$ can be extended to $\mathrm{SO}_{2}$ gas by comparison of spectral absorption coefficients at $8623 \mathrm{~nm}$ $8628 \mathrm{~nm}$. This analysis results in $3 \sigma \mathrm{MDL}$ for $\mathrm{SO}_{2}$ to be calculated as $25 \mathrm{ppm}$ for $\mathrm{R}_{1 f}, 49 \mathrm{ppm}$ for $\mathrm{R}_{2 f}$ and $65 \mathrm{ppm}$ for $\mathrm{R}_{2 f} / \mathrm{R}_{1 f}$ techniques at 0.1 bar pressure.

Although the data presented in this paper uses a specific polymer, that has only been tested at wavelengths larger than $8000 \mathrm{~nm}$, there is also preliminary data showing similar effects for another polymer type (EnvisionTec R11) at wavelengths around $5.2 \mu \mathrm{m}$.

In comparison with QEPAS techniques, the overall sensitivity of the $R_{2 f} / R_{1 f}$ measurement is lower due to its low SNR and Q-factor which leads to a slightly reduced noise immunity. In QEPAS, to contain and amplify the acoustic signals, micro-resonators placed in between quartz tuning forks are commonly used. The use of a micro-resonator made out of a polymer absorbing at the target wavelength range could combine the here presented calibration-free technique and the high-sensitivity of QEPAS into one highly efficient technique

\section{CONCLUSION}

This paper outlined an alternative solution to a major issue of PAS gas sensors in their diverse deployment in industrial uses; the need for continuous calibration. The proposed solution is the adaptation of the $R_{2 f} / R_{1 f}$ calibration technique into a WMS-PAS detection scheme. The technique exploits a DClevel called CDAS in if signals, not commonly observed in PAS signals, to normalize $2 \mathrm{f}$ signal against unexpected changes in the system. The CDAS baseline for the PAS signal was created through a targeted laser excitation interaction with the inner walls of the acoustic resonator cell. The changes in gas concentration and optical light input into the cell was shown to be calibrated successfully between $0 \%$ and $100 \% \mathrm{CH}_{4}$ and up to $78.3 \%$ attenuation respectively. The normalization for changes in other factors affecting the gas such as pressure and temperature can also be achieved, but it is out of scope of this paper.
This new technique provides all the advantages of TDLSWMS detection in terms of versatility of use as well as well known advantages of PAS, such as miniaturization and use of cost-effective components and improved performance at longer wavelengths where commercial uncooled photodetectors have reduced sensitivity. Overall, the developed technique of $R_{2 f} / R_{1 f}$ calibration for WMS-PAS may enable the practical application of PAS outside laboratories, in a multitude of real-world applications.

\section{REFERENCES}

[1] A. Elia, P. M. Lugara, C. D. Franco, and V. Spagnolo. Photoacoustic Techniques for Trace Gas Sensing Based on Semiconductor Laser Sources. Sensors, 9(12):9616-9628, 2009.

[2] T. Berer, M. Brandstetter, A. Hochreiner, G. Langer, W. Märzinger P. Burgholzer, and B. Lendl. Remote mid-infrared photoacoustic spectroscopy with a quantum cascade laser. Opt. Lett., 40(15):34763479, 2015.

[3] A. Schwaighofer, M. Brandstetter, and B. Lendl. Quantum cascade lasers (QCLs) in biomedical spectroscopy. Chem. Soc. Rev., 46(19):59035924, 2017.

[4] J. S. Li, B. Yu, H. Fischer, W. Chen, and A. P. Yalin. Contributed review: quantum cascade laser based photoacoustic detection of explosives. Rev Sci Instrum., 86(3):031501, 2015.

[5] J. S. Li, C. Weidong, and B. Yu. Recent Progress on Infrared Photoacoustic Spectroscopy Techniques. Appl. Spectrosc. Rev., 46(6):440-471, 2011.

[6] J. Hodgkinson and R. P. Tatam. Optical gas sensing: a review. Meas. Sci. Technol., 24(1):012004, 2013.

[7] A. Miklós, S. Schäfer, and P. Hess. Photoacoustic spectroscopy, theory. In John C. Lindon, editor, Encyclopedia of Spectroscopy and Spectrometry, pages 1815-1822. Elsevier, 1999.

[8] S. Schilt, L. Thevenaz, and P. Robert. Wavelength modulation spectroscopy: combined frequency and intensity laser modulation. Appl. Opt., 42(33):6728-6738, 2003.

[9] K . Duffin, A. J. McGettrick, W. Johnstone, G. Stewart, and D. G. Moodie. Tunable diode-laser spectroscopy with wavelength modulation: A calibration-free approach to the recovery of absolute gas absorption line shapes. J. Light. Technol, 25(10):3114-3125, 2007.

[10] A. Miklós, P. Hess, and Z. Bozóki. Application of acoustic resonators in photoacoustic trace gas analysis and metrology. Rev. Sci. Instrum., 72(4):1937-1955, 2001.

[11] P. Patimisco, G. Scamarcio, F. K. Tittel, and V. Spagnolo. QuartzEnhanced Photoacoustic Spectroscopy: A Review. Sensors, 14(4):61656206, 2014.

[12] L. Xiong, W. Bai, F. Chen, X. Zhao, F. Yu, and G. J. Diebold. Photoacoustic trace detection of gases at the parts-per-quadrillion level with a moving optical grating gas detection with a moving photoacoustic source. Proc. Natl. Acad. Sci. U.S.A., 114(28):7246-7249, 2017.

[13] H.A. Beck, R Niessner, and C. Haisch. Development and characterization of a mobile photoacoustic sensor for on-line soot emission monitoring in diesel exhaust gas. Anal. Bioanal. Chem., 375(8):1136-1143, 2003.

[14] P. L. Meyer and M. W. Sigrist. Atmospheric pollution monitoring using CO2-laser photoacoustic spectroscopy and other techniques. Rev. Sci. Instrum., 61(7):1779-1807, 1990.

[15] R. Bartlome, M. Kaučikas, and M. W. Sigrist. Modulated resonant versus pulsed resonant photoacoustics in trace gas detection. Appl. Phys. B, 96(2-3):561-566, 2009.

[16] M. Suchenek and T. Starecki. Pulse measurements of the frequency response of a photoacoustic cell. Int. J. Thermophys., 32(4):893-900, 2011.

[17] M. Szakall, A. Varga, A. Pogany, Z. Bozoki, and G. Szabo. Novel resonance profiling and tracking method for photoacoustic measurements. Appl. Phys. B, 94(4):691-698, 2009.

[18] J. Huber, A. Ambs, and J. Wöllenstein. Miniaturized Photoacoustic Carbon Dioxide Sensor with Integrated Temperature Compensation for Room Climate Monitoring. Procedia Eng., 120:283-288, 2015.

[19] P. L. Meyer and M. W. Sigrist. Atmospheric pollution monitoring using $\mathrm{CO}_{2}$-laser photoacoustic spectroscopy and other techniques. Rev. Sci. Instrum., 61(7):1779-1807, 1990. 
[20] J. Saarela, T. Sorvajärvi, T. Laurila, and J. Toivonen. Phase-sensitive method for background-compensated photoacoustic detection of $\mathrm{NO} 2$ using high-power LEDs. Opt. Express, 19(S4):A725-A732, 2011.

[21] A. A. Kosterev, Y.A. Bakhirk, F.K. Tittel, S. Blaser, Y. Bonetti, and L. Hvozdara. Photoacoustic phase shift as a chemically selective spectroscopic parameter. Appl. Phys. B, 78(6):673-676, 2004.

[22] H. Wu, L. Dong, H. Zheng, Y. Yu, W. Ma, L. Zhang, W. Yin, L. Xiao, S. Jia, and F. K. Tittel. Beat frequency quartz-enhanced photoacoustic spectroscopy for fast and calibration-free continuous trace-gas monitoring. Nat. Commun., 8(15331), 2017.

[23] G. B. Rieker, J. B. Jeffries, and R. K. Hanson. Calibration-free wavelength-modulation spectroscopy for measurements of gas temperature and concentration in harsh environments. Appl. Opt., 48(29):5546$5560,2009$.

[24] R. Bauer, G. Stewart, W. Johnstone, E. Boyd, and M. Lengden. 3d printed miniature gas cell for photoacoustic spectroscopy of trace gases. Opt. Lett., 39(16):4796-4799, 2014.

[25] R. Bauer, T. Legg, D. Mitchell, G. M. H. Flockhart, G. Stewart, W. Johnstone, and M. Lengden. Miniaturized photoacoustic trace gas sensing using a raman fiber amplifier. J. Lightwave Technol., 33(18):3773-3780, 2015

[26] J. Reid and D. Labrie. Second-harmonic detection with tunable diode lasers-Comparison of experiment and theory. Appl. Phys. B, 26(3):203-210, 1981.

[27] A. J. McGettrick, K. Duffin, W. Johnstone, G. Stewart, and D. G. Moodie. Tunable Diode Laser Spectroscopy With Wavelength Modulation: A Phasor Decomposition Method for Calibration-Free Measurements of Gas Concentration and Pressure. J. Lightwave Technol., 26(4):432-440, 2008 